\title{
Surgical Management of a Locally Advanced Symptomatic Recurrence of Penile Sarcoma Secondary to Prostate Brachytherapy
}

\author{
Tariq S. Hakky, Patrick Espiritu, Alejandro R. Rodriguez, Nicholas Gould, Philippe E. Spiess \\ Department of Genitourinary Oncology, Moffitt Cancer Center and Department of Urology, University of \\ South Florida, Tampa, Florida, USA
}

\begin{abstract}
Background: The surgical management of patients with symptomatic metastatic or locally advanced recurrences involving the penis remains poorly characterized. The aim of the present abstract and video is to detail our experience in the surgical management of a specific patient with a locally advanced symptomatic recurrence of penile sarcoma secondary to prostate cancer treated with primary brachytherapy.

Materials and Methods: A 70 year old male patient initially treated for localized prostate cancer with interstitial brachytherapy at an outside facility developed an unfortunate secondary malignancy consisting of a locally advanced penile sarcoma involving as well the prostate and base of the bladder. Despite our best efforts to control his pain, he developed a very symptomatic local recurrence with a secondary penile abscess and purulent periurethral drainage. At this time, it was felt a surgical resection consisting of a total penectomy, urethrectomy, cystoprostatectomy, and ileal conduit urinary diversion would be the best option for local cancer control in this particular patient.

Results: The patient underwent the surgical resection without any complications as illustrated in this surgical video, with a jejunal intestinal mass identified at the time of surgery which was resected with a primary bowel anastomosis performed. The patient was discharged from hospital uneventfully with his symptomatic local recurrence being successfully managed and the patient no longer requiring oral narcotics for pain control. The pathological report confirmed a locally advanced sarcoma involving the penile, prostate, and bladder which was resected with negative surgical margins and the jejunal mass was confirmed to represent a small bowel sarcoma metastatic site.

Conclusion: As highlighted in the present video, the treatment of a symptomatic sarcoma local recurrence contiguously involving the penis can be successfully managed provided the patient is informed of the potential morbidity and psychosocial implications imparted by performing a total penectomy and adjacent organ resection.
\end{abstract}

\section{ARTICLE INFO}

Available at: www.brazjurol.com.br/videos/march_april_2013/Hakky_293_294video.htm

Int Braz J Urol. 2013; 39 (Video \#5): 293-4

Submitted for publication:

December 01, 2012

Correspondence address:

Dr. Philippe E. Spiess,

Associate Professor

Department of Genitourinary Oncology

Moffitt Cancer Center

Accepted after revision:

Tampa, FL, USA

January 30, 2013

E-mail: philippe.spiess@moffitt.org 


\section{EDITORIAL COMMENT}

Radiation therapy for prostate cancer can be an effective treatment but does have its complications. In this very well done video the authors demonstrate their surgical technique for the management of a secondary penile sarcoma following brachytherapy for low-grade prostate cancer. A radical penectomy with vertical rectus myocutaneous flap in addition to radical cystoprostatectomy and ileal conduit was performed.
This was an impressive display of surgical skill for this rare but potentially devastating problem. I felt that the video was of very high quality and brings to light the difficult decisions which are needed in such a case. Although the video does suggest that the patient is free of recurrence clearly these highly aggressive tumor types are at high risk of recurrence with short to intermediate follow-up.

Dr. A. Karim Kader Associate Professor, Department of Urology University of San Diego San Diego, CA, USA

E-mail: jmthompson@ucsd.edu 